\title{
Curvature Correlograms for Content Based Retrieval of 3D Objects
}

\author{
G. Antini, S. Berretti, A. Del Bimbo, and P. Pala \\ Dipartimento di Sistemi e Informatica, \\ Università degli Studi di Firenze, \\ via S.Marta 3, 50139 Firenze, Italy \\ \{antini, berretti, delbimbo, pala\}@dsi.unifi.it
}

\begin{abstract}
Along with images and videos, 3D models have raised a certain interest for a number of reasons, including advancements in 3D hardware and software technologies, their ever decreasing prices and increasing availability, affordable 3D authoring tools, and the establishment of open standards for 3D data interchange. The resulting proliferation of 3D models demands for tools supporting their effective and efficient management, including archival and retrieval.

In order to support effective retrieval by content of 3D objects and enable retrieval by object parts, information about local object structure should be combined with spatial information on object surface. In this paper, as a solution to this requirement, we present a method relying on curvature correlograms to perform description and retrieval by content of 3D objects.

Experimental results are presented both to show results of sample queries by content and to compare-in terms of precision/recall figures- the proposed solution to alternative techniques.
\end{abstract}

\section{Introduction}

Beside image and video databases, archives of 3D models have recently gained increasing attention for a number of reasons: advancements in 3D hardware and software technologies, their ever increasing availability at affordable costs, and the establishment of open standards for 3D data interchange (e.g. VRML, X3D).

Three-dimensional acquisition of a real-world object, capturing both object geometry and its visual features (surface color and texture), can be achieved through many different techniques, including CAD, 3D laser scanners, structured light systems and photogrammetry. Thanks to the availability of these technologies, 3D models are being created and employed in a wide range of application domains, including medicine, computer aided design and engineering, and cultural heritage.

In this framework the development of techniques to enable retrieval by content of 3D models assumes an ever increasing relevance. This is particularly the case in the fields of cultural heritage and historical relics, where there is a growing interest in solutions enabling preservation of relevant artworks (e.g. vases, sculptures, and handicrafts) as well as cataloguing and retrieval by content. In these fields, retrieval by content can be employed to detect commonalities between 3D objects (e.g. the "signature" of the artist) or to monitor the temporal evolution of a defect (e.g. the amount of bending for wooden tables). 


\subsection{Previous Work}

Methods addressing retrieval of 3D models can be distinguished based on different aspects, such as the type of representation used for geometry, the use of information about models' appearance (i.e. colour and/or texture), the need for manual annotation.

Generally, two broad classes of approaches can be distinguished: view-based and structure-based. In the former, salient features of an object are extracted from a set of $2 \mathrm{D}$ views of the object itself. In the latter, object features are computed directly in the three-dimensional space in order to capture prominent characteristics of the object structure.

Description and retrieval of 3D objects based on description and retrieval of 2D views has been addressed in [1] and [2]. However, the effectiveness of these solutions is limited to description and retrieval of simple objects. In fact, as complex objects are considered, occlusions prevent to capture distinguishing 3D features using 2D views.

Recently, a hybrid approach — that is not entirely view-based or structure-basedhas been proposed in [9] relying on the use of spin images for content description and matching. Description of 3D structure for the purpose of recognition or retrieval has been addressed for some time. A few authors have investigated analytical 3D models, but this is not always a viable solution, as there are many limitations in providing parameterizations of arbitrary models. In [3] retrieval of 3D objects based on similarity of surface segments is addressed. Surface segments model potential docking sites of molecular structures.

Much attention has been recently devoted to free-form (i.e. polygonal) meshes. The system developed within the Nefertiti project supports retrieval of 3D models based on both geometry and appearance (i.e. colour and texture) [4]. Also Kolonias et al. have used dimensions of the bounding box (i.e. its aspect ratios) and a binary voxel-based representation of geometry [5]. They further relied on a third feature, namely a set of paths, outlining the shape (model routes). In [6] a method is proposed to select feature points which relies on the evaluation of Gaussian and median curvature maxima, as well as of torsion maxima on the surface. In [7], Elad et al. use moments (up to the 4-7th order) of surface points as basic features to support retrieval of 3D models. Differently from the case of 2D images, evaluation of moments is not affected by (self-)occlusions.

In order to capture geometric features as well as their arrangement on the object surface, in [8] description and retrieval of 3D objects is accomplished through a combination of warping and projection. However, this method can be applied only to objects whose surface defines the boundary of a simply connected 3D region. Moreover, warping may introduce irregular deformation of the object surface before its projection on a 2D map.

Correlograms have been previously used with success for retrieval of images based on color content [14]. In particular, with respect to description based on histograms of local features, correlograms enable also encoding of information about the relative localization of local features. In [15], histograms of surface curvature have been used to support description and retrieval of $3 \mathrm{D}$ objects. However, since histograms do not include any spatial information, the system is liable to false positives.

In this paper, we present a model for representation and retrieval of $3 \mathrm{D}$ objects based on curvature correlograms. Correlograms are used to encode information about curva- 
ture values and their localization on the object surface. For this peculiarity, description of $3 \mathrm{D}$ objects based on correlograms of curvature proves to be very effective for the purpose of content based retrieval of 3D objects.

This paper is organized as follows: in Sect 2 representation of object structure through curvature correlograms is presented; in Sect 3 some distance measures are defined to be used for computing the similarity between two curvature correlograms; finally, in Sect 4 experimental results and performance comparison with alternative approaches are presented.

\section{Computation of Curvature Correlograms}

High resolution 3D models obtained through scanning of real world objects are often affected by high frequency noise, due to either the scanning device or the subsequent registration process. Hence, smoothing is required to cope with such models for the purpose of extracting their salient features. This is especially true if salient features are related to differential properties of mesh surface (e.g. surface curvature).

Selection of a smoothing filter is a critical step, as application of some filters entails changes in the shape of the models. In the proposed solution, we adopted the filter first proposed by Taubin [10]. This filter, also known as $\lambda \mid \mu$ filter, operates iteratively, and interleaves a Laplacian smoothing weighed by $\lambda$ with a second smoothing weighed with a negative factor $\mu(\lambda>0, \mu<-\lambda<0)$. This second step is introduced to preserve the model's original shape.

Let $\mathcal{M}$ be a mesh. We denote with $E, V$ e $F$, the sets of all edges, vertices and faces of the mesh. With $N_{V}, N_{E}$ and $N_{F}$, we denote the cardinality of sets $V, E$ and $F$.

Given a vertex $v \in \mathcal{M}$, the principal curvature of $\mathcal{M}$ at vertex $v$ is indicated as $k_{1}(v)$ and $k_{2}(v)$. The mean curvature $\bar{k}_{v}$ is related to the principal curvature $k_{1}(v)$ and $k_{2}(v)$ by the equation:

$$
\bar{k}_{v}=\frac{k_{1}(v)+k_{2}(v)}{2}
$$

Details about computation of the principal and mean curvature for a mesh can be found in [11].

Values of the mean curvature are quantized into $2 N+1$ classes of discrete values. For this purpose, a quantization module processes the mean curvature value through a stair-step function so that many neighboring values are mapped to one output value:

$$
\mathcal{Q}(\bar{k})= \begin{cases}N \Delta & \text { if } \bar{k} \geq N \Delta \\ i \Delta & \text { if } \bar{k} \in[i \Delta,(i+1) \Delta) \\ -i \Delta & \text { if } \bar{k} \in[-(i+1) \Delta,-i \Delta) \\ -N \Delta & \text { if } \bar{k} \leq-N \Delta\end{cases}
$$

with $i \in\{0, \ldots, N-1\}$ and $\Delta$ a suitable quantization parameter (in the experiments reported in $\operatorname{Sect} 4 N=100$ and $\Delta=0.15)$. Function $\mathcal{Q}(\cdot)$ quantize values of $\bar{k}$ into $2 N+1$ distinct classes $\left\{c_{i}\right\}_{i=-N}^{N}$.

To simplify notation, $v \in \mathcal{M}_{i}$ is synonymous with $v \in \mathcal{M}$ and $\mathcal{Q}\left(\bar{k}_{v}\right)=c_{i}$. 
Definition 1 (Histogram of Curvature). Given a quantization of curvature values into $2 N+1$ classes $\left\{c_{i}\right\}_{i=-N}^{N}$, the histogram of curvature $h_{c_{i}}(\mathcal{M})$ of the mesh $\mathcal{M}$ is defined as:

$$
h_{c_{i}}(\mathcal{M})=N_{V} \cdot \operatorname{Pr}_{v_{i} \in \mathcal{M}}\left[v_{i} \in \mathcal{M}_{i}\right]
$$

being $N_{V}$ the number of mesh vertices.

In doing so, $h_{c_{i}}(\mathcal{M}) / N_{V}$ is the probability that the quantized curvature of a generic vertex of the mesh belongs to classes $c_{i}$.

The correlogram of curvature is defined with respect to a predefined distance value $\delta$. In particular, the curvature correlogram $\gamma_{c_{i} c_{j}}^{(\delta)}$ of a mesh $\mathcal{M}$ is defined as:

$$
\gamma_{c_{i}, c_{j}}^{(\delta)}(\mathcal{M})=\operatorname{Pr}_{v_{1}, v_{2} \in \mathcal{M}}\left[\left(v_{1} \in \mathcal{M}_{c_{i}}, v_{2} \in \mathcal{M}_{c_{j}}\right) \mid\left\|v_{1}-v_{2}\right\|=\delta\right]
$$

In this way, $\gamma_{c_{i}, c_{j}}^{(\delta)}(\mathcal{M})$ is the probability that two vertices that are $\delta$ far away from each other have curvature belonging to class $c_{i}$ and $c_{j}$, respectively.

Ideally, $\left\|v_{1}-v_{2}\right\|$ should be the geodesic distance between vertices $v_{1}$ and $v_{2}$. However, this can be approximated with the $k$-ring distance if the mesh $\mathcal{M}$ is regular and triangulated [12].

Definition 2 (1-ring). Given a generic vertex $v_{i} \in \mathcal{M}$, the neighborhood or 1-ring of $v_{i}$ is the set:

$$
V^{v_{i}}=\left\{v_{j} \in \mathcal{M}: \exists e_{i j} \in E\right\}
$$

being $E$ the set of all mesh edges (if $e_{i j} \in E$ there is an edge that links vertices $v_{i}$ and $v_{j}$ ).

The set $V^{v_{i}}$ can be easily computed using the morphological operator dilate [13]:

$$
V^{v_{i}}=\operatorname{dilate}\left(v_{i}\right)
$$

Through the dilate operator, the concept of 1-ring can be used to define, recursively, generic $k^{\text {th }}$ order neighborhood:

$$
\text { ring }_{k}=\text { dilate }^{k} \cap \text { dilate }^{k-1}
$$

Definition of $k^{t h}$ order neighborhood enables definition of a true metric between vertices of a mesh. This metric can be used for the purpose of computing curvature correlograms as an approximation of the usual geodesic distance (that is computationally much more demanding). According to this, we define the $k$-ring distance between two mesh vertices as $d_{\text {ring }}\left(v_{1}, v_{2}\right)=k$ if $v_{2} \in \operatorname{ring}_{k}\left(v_{1}\right)$.

Function $d_{\text {ring }}\left(v_{1}, v_{2}\right)=k$ is a true metric, in fact:

1. $d_{\text {ring }}(u, v) \geq 0$, and $d_{\text {ring }}(u, v)=0$ if and only if $u=v$.

2. $d_{\text {ring }}(u, v)=d_{\text {ring }}(v, u)$

3. $\forall w \in \mathcal{M} d(u, v) \leq d(u, w)+d(w, v)$

Based on the $d_{\text {ring }}(\cdot)$ distance, the correlogram of curvature can be redefined as follows:

$$
\gamma_{c_{i}, c_{j}}^{(k)}(\mathcal{M})=\operatorname{Pr}_{v_{1}, v_{2} \in \mathcal{M}}\left[\left(v_{1} \in \mathcal{M}_{c_{i}}, v_{2} \in \mathcal{M}_{c_{j}}\right) \mid d_{\text {ring }}\left(v_{1}, v_{2}\right)=k\right]
$$

Figs 1(a)-(b) show the correlograms of three models derived from two different model categories, statue and dinosaur, respectively. 
(a)

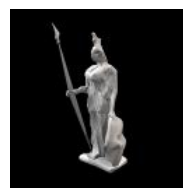

(b)

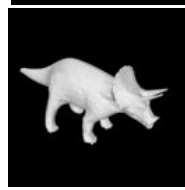

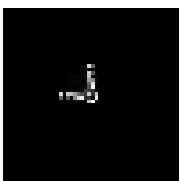

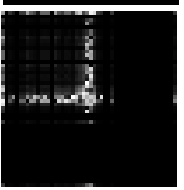

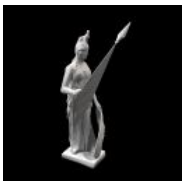

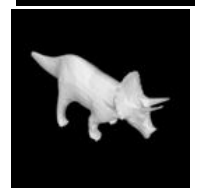

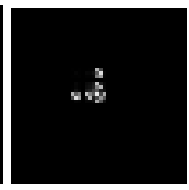
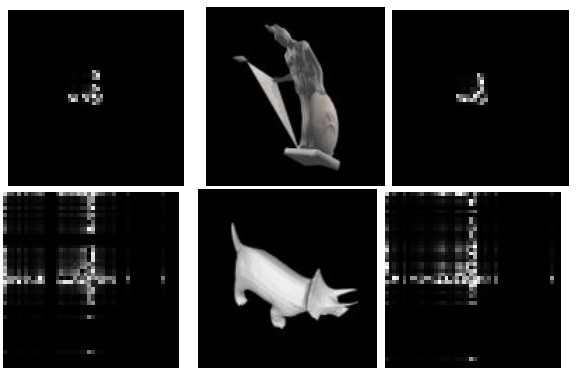

Fig. 1. In (a) and (b) correlograms of three models, taken from two distinct categories, namely statue and dinosaur, are shown.

\section{Matching Curvature Correlograms}

Several distance measures have been proposed to compute the dissimilarity of distribution functions. In order to compute the similarity between curvature correlograms of two distinct meshes $\gamma_{c_{i}, c_{j}}^{(k)}\left(\mathcal{M}_{1}\right)$ and $\gamma_{c_{i}, c_{j}}^{(k)}\left(\mathcal{M}_{2}\right)$ we experimented the following distance measures:

Minkowsky-form distance

$$
d_{\mathcal{L}_{p}}=\left[\sum_{i, j=-N}^{N}\left|\gamma_{c_{i}, c_{j}}^{(k)}\left(\mathcal{M}_{1}\right)-\gamma_{c_{i}, c_{j}}^{(k)}\left(\mathcal{M}_{2}\right)\right|^{p}\right]^{1 / p}
$$

Histogram intersection

$$
d_{H I}=1-\frac{\sum_{i, j=-N}^{N} \min \left(\gamma_{c_{i}, c_{j}}^{(k)}\left(\mathcal{M}_{1}\right), \gamma_{c_{i}, c_{j}}^{(k)}\left(\mathcal{M}_{2}\right)\right)}{\sum_{i, j=-N}^{N} \gamma_{c_{i}, c_{j}}^{(k)}\left(\mathcal{M}_{2}\right)}
$$

$\chi^{2}$-statistics

$$
d_{\chi^{2}}=\sum_{i, j=-N}^{N} \frac{\left(\gamma_{c_{i}, c_{j}}^{(k)}\left(\mathcal{M}_{1}\right)-\gamma_{c_{i}, c_{j}}^{(k)}\left(\mathcal{M}_{2}\right)\right)^{2}}{2\left(\gamma_{c_{i}, c_{j}}^{(k)}\left(\mathcal{M}_{1}\right)+\gamma_{c_{i}, c_{j}}^{(k)}\left(\mathcal{M}_{2}\right)\right)}
$$

Kullback-Leibler divergence

$$
d_{K L}=\sum_{i, j=-N}^{N} \gamma_{c_{i}, c_{j}}^{(k)}\left(\mathcal{M}_{1}\right) \log \frac{\gamma_{c_{i}, c_{j}}^{(k)}\left(\mathcal{M}_{1}\right)}{\gamma_{c_{i}, c_{j}}^{(k)}\left(\mathcal{M}_{2}\right)}
$$

Using a groundtruth database, the above distance measures have been compared in terms of precision and recall figures. Results of this analysis (not reported in this paper for lack of space) suggest that the best performance is achieved by using $\chi^{2}$-statistics to measure the distance between curvature correlograms.

\section{Experimental Results}

Approximately 300 models were collected to build the test database. These comprise four classes of models: taken from the web, manually authored (with a 3D CAD soft- 


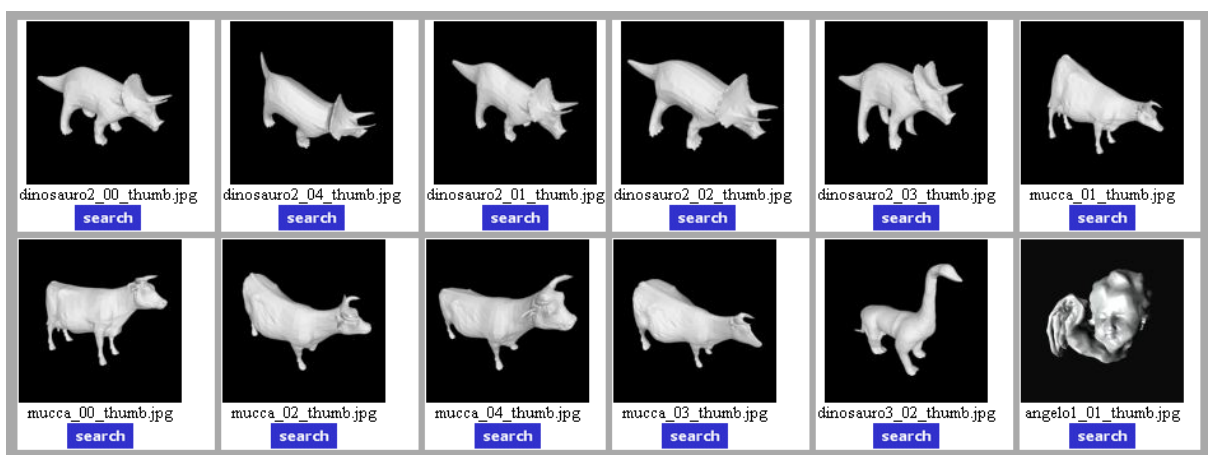

Fig. 2. A retrieval example, using the model of a dinosaur as the query. Other models of dinosaurs were retrieved first, followed by models of other objects which display similar features.

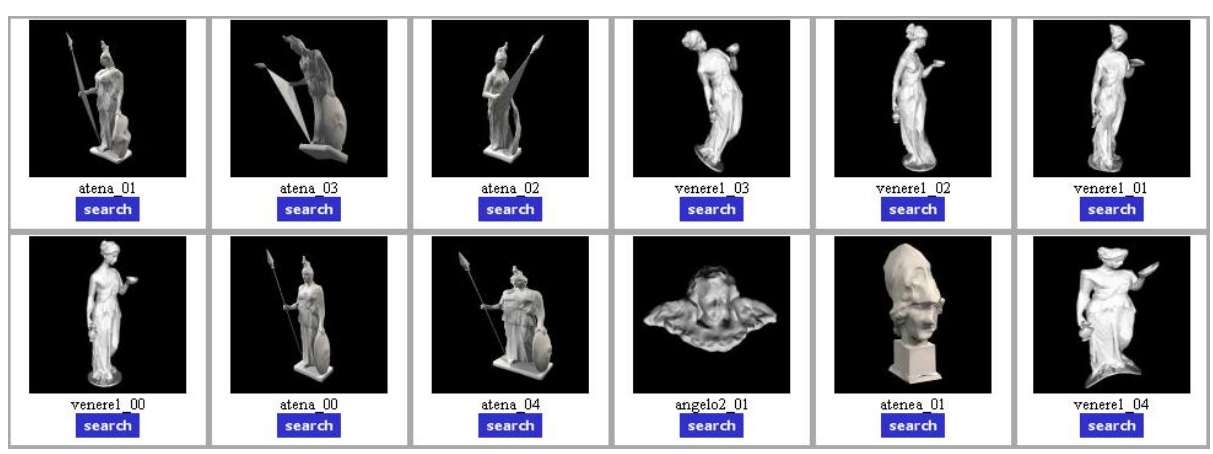

Fig. 3. The model of a statue is used as query. Other models of the same statue are retrieved, as well as models which display busts or different statues.

ware), high quality 3D scans from the De Espona 3D Models Encyclopedia1, and variations of the previous three classes (obtained through deformation or application of noise, which caused points surface to be moved from their original locations). Content descriptors, in the form of curvature correlograms, were then extracted from database objects and added to the index.

Fig 2 shows a retrieval example where the model of a dinosaur is used as a query. The result set displays all models of similar animals in the first positions. These animals all share some major features such as four legs, a tail and two horns.

Retrieval results for a query representing a statue are shown in Fig 3 . In this case, models of statues and busts are ranked in the first positions of the retrieval set.

In Fig. 4 the precision-recall curve of the proposed approach is compared with the curve of alternative approaches. In particular, approaches based on $3 \mathrm{D}$ geometric moments [7], curvature histograms [15], curvature maps [8] and spin images [9] are considered. The proposed solution shows an improved performance with respect to all the four alternative approaches.

\footnotetext{
${ }^{1}$ http: / / www . deespona.com
} 


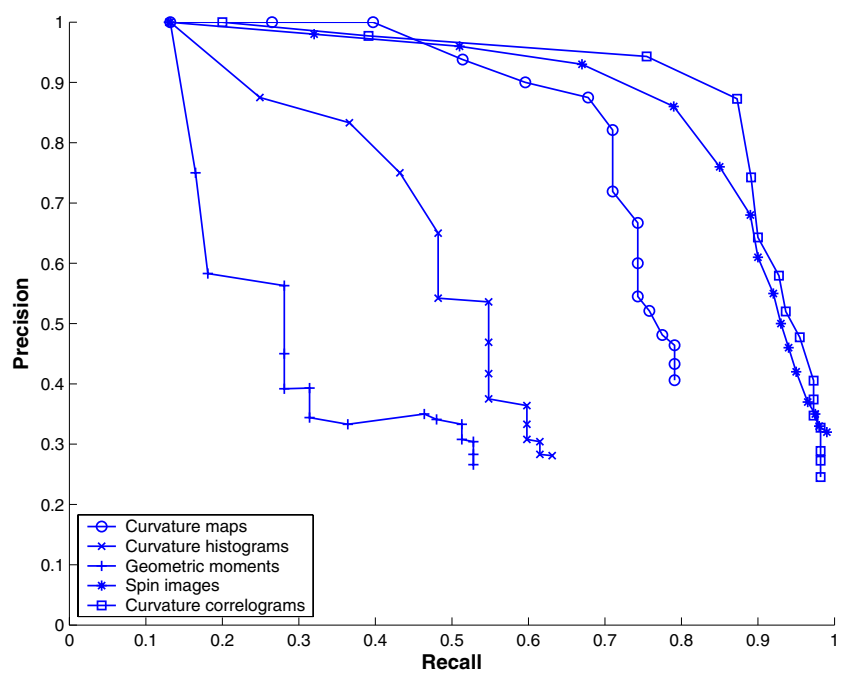

Fig. 4. The average Precision-Recall curves over a given set of queries show an improved performance of the correlograms with respect to four alternative approaches.

\section{Conclusions and Future Work}

In this paper we have presented an approach to retrieval by content of $3 \mathrm{D}$ objects based on curvature correlograms. The main advantage of correlograms relates to their ability to encode not only distribution of features but also their arrangement on the object surface. Experimental results have shown that the proposed solution is well suited for the purpose of retrieval. Furthermore, results showed that the approach performs better than previous approaches to content-based retrieval of $3 \mathrm{D}$ objects. Future work will address extension of correlograms to deal with multiresolution descriptors as well as the definition of suitable distance measures to cope with retrieval by object parts.

\section{References}

1. S. Mahmoudi, M. Daoudi. "3D models retrieval by using characteristic views," in Proc. of 16th Int'l Conf. on Pattern Recognition, Vol.2, pp.457-460, 11-15 Aug, 2002.

2. R. Ohbuchi, M. Nakazawa, T. Takei. "Retrieving 3D Shapes based on Their Appearance," in Proc. of MIR'03, Berkeley, CA, USA, Nov. 2003, pp.39-46.

3. H.P. Kriegel, T. Seidl. "Approximation-Based Similarity Search for 3D Surface Segments," GeoInformatica Journal, 2(2):113-147, Kluwer Academic Publisher, 1998.

4. E. Paquet, M. Rioux. "Nefertiti: a query by content system for three-dimensional model and image database management," Image Vision and Computing, 17(2):157-166, 1999.

5. I. Kolonias, D. Tzovaras, S. Malassiotis, M. G. Strintzis, "Content-Based Similarity Search of VRML Models Using Shape Descriptors", in Proc. of International Workshop on ContentBased Multimedia Indexing, Brescia (I), September 19-21, 2001. 
6. F. Mokhtarian, N. Khalili, P. Yeun. "Multi-scale free-form 3D object recognition using 3D models," Image and Vision Computing, 19(5):271-281, 2001.

7. M. Elad, A. Tal, S. Ar. "Content Based Retrieval of VRML Objects - An Iterative and Interactive Approach," EG Multimedia, September 2001, 97-108.

8. J. Assfalg, A. Del Bimbo, P. Pala. "Curvature Maps for 3D CBR”, in Proc. of Int'l Conf. on Multimedia and Expo (ICME'03), Baltimore (MD), July 2003.

9. J. Assfalg, G. D’Amico, A. Del Bimbo, P. Pala. "3D content-based retrieval with spin images," in Proc. of Int'l Conf. on Multimedia and Expo (ICME'04), Taipei, Taiwan, June 2730, 2004.

10. G. Taubin. "A Signal Processing Approach to Fair Surface Design," Computer Graphics (Annual Conference Series), 29:351-358, 1995.

11. G. Taubin. "Estimating the Tensor of Curvature of a Surface from a Polyhedral Approximation," in Proc. of Fifth International Conference on Computer Vision (ICCV'95), pp.902-907.

12. Mathieu Desbrun, Mark Meyer, Peter Schroder and Alan H. Barr. "Discrete DifferentialGeometry Operators in $n$ D”, Caltech, 2000.

13. Christian Rössl, Leif Kobbelt, Hans-Peter Seidel. "Extraction of Feature Lines on Triangulated Surfaces using Morphological Operators," in Smart Graphics, Proceedings of the 2000 AAAI Symposium

14. J. Huang, R. Kumar, M. Mitra, W.-J. Zhu, R. Zabih. "Statial Color Indexing and Application," in Internation Journal of Computer Vision, Vol. 35, pp. 245-268, 1999

15. G. Hetzel, B. Leibe, P. Levi, B. Schiele. "3D Object Recognition from Range Images using Local Feature Histograms," in Proc. of Int. Conf. on Computer Vision and Pattern Recognition (CVPR'01), Kauai Marriott, Hawaii, Dec. 9-14, 2001. 